University of Nebraska - Lincoln

DigitalCommons@University of Nebraska - Lincoln

Faculty Publications, Department of Psychology

Psychology, Department of

$6-2012$

\title{
Communication-Based Assessment of Developmental Age for Young Children with Developmental Disabilities
}

\author{
Shari L. DeVeney \\ University of Nebraska-Lincoln, sharideveney@gmail.com \\ Lesa Hoffman \\ University of Nebraska-Lincoln, Ihoffman2@unl.edu \\ Cynthia J. Cress \\ University of Nebraska-Lincoln, ccress1@unl.edu
}

Follow this and additional works at: https://digitalcommons.unl.edu/psychfacpub

Part of the Psychiatry and Psychology Commons

DeVeney, Shari L.; Hoffman, Lesa; and Cress, Cynthia J., "Communication-Based Assessment of Developmental Age for Young Children with Developmental Disabilities" (2012). Faculty Publications, Department of Psychology. 574.

https://digitalcommons.unl.edu/psychfacpub/574

This Article is brought to you for free and open access by the Psychology, Department of at DigitalCommons@University of Nebraska - Lincoln. It has been accepted for inclusion in Faculty Publications, Department of Psychology by an authorized administrator of DigitalCommons@University of Nebraska - Lincoln. 


\title{
Communication-Based Assessment of Developmental Age for Young Children with Developmental Disabilities
}

\author{
Shari L. DeVeney, Lesa Hoffman, and Cynthia J. Cress \\ University of Nebraska-Lincoln \\ Corresponding author — Shari L. DeVeney, email sharideveney@gmail.com
}

\begin{abstract}
Purpose: In this study, the authors compared a multiple-domain strategy for assessing developmental age of young children with developmental disabilities who were at risk for long-term reliance on augmentative and alternative communication (AAC) with a communication-based strategy composed of receptive language and communication indices that may be less affected by physically challenging tasks than traditional developmental age scores.

Method: Participants were 42 children (age 9-27 months) with developmental disabilities and who were at risk for long-term reliance on AAC. Children were assessed longitudinally in their homes at 3 occasions over 18 months using multiple-domain and communication-based measures. Confirmatory factor analysis examined dimensionality across the measures, and age-equivalence scores under each strategy were compared, where possible.

Results: The communication-based latent factor of developmental age demonstrated good reliability and was almost perfectly correlated with the multiple-domain latent factor. However, the mean age-equivalence score of the communication-based assessment significantly exceeded that of the multiple-domain assessment by 5.3 months across ages.

Conclusions: Clinicians working with young children with developmental disabilities should consider a communication-based approach as an alternative developmental age assessment strategy for characterizing children's capabilities, identifying challenges, and developing interventions. A communication-based developmental age estimation is sufficiently reliable and may result in more valid inferences about developmental age for children whose developmental or cognitive age scores may otherwise be limited by their physical capabilities.
\end{abstract}

Keywords: infants and toddlers, cognition and language, developmental disorders, people with severe disabilities, augmentative and alternative communication

$\mathrm{C}$ hildren with developmental disabilities are expected to show variability from typical expectations in both the types and rates of skill development in early childhood. One challenge for practitioners is to determine how to characterize these variable skills in ways that are both reliable and representative of a child's skills and disabilities. For children with developmental disabilities, speech-language pathologists need to consider a child's communication skills and potential in reference to his or her developmental skills and impairments in other domains. A comprehensive assessment in early childhood should include a variety of dynamic, curriculum-based, family-based, and performance assessments across domains (Meisels \& Atkins-Burnett, 2000). It is important to note that different methods may be needed for assessing domains of impairment and verifying the need for related services than would be necessary for assessing a child's developmental and communicative potential in order to plan interventions and predict outcomes based on a child's domains of strength. When the goal of assessment relates to determining family goals or planning interventions, clinicians tend to rely more heavily on open-ended interviews and dynamic observations that place a higher demand on the skill of the assessor to achieve results that are reliable and representative of the child's abilities (Greenwood \& Carta, 2010). When the goal of assessment instead relates to diagnosis or prediction of outcomes based on discrete skill estimates, clinicians tend to rely more on quantifiable behavior probes that depend on the appropriate match of a standardized assessment task to a child's capability across domains to achieve representative estimates of a child's abilities. 
Estimates of developmental age are commonly used to characterize and predict skills in children with developmental disabilities, based on a child's response to standardized multiple-domain probes relative to expected responses of typically developing peers. Developmental age has been used by interdisciplinary assessment teams to identify impairment, characterize patterns of skills and deficits, predict skill development over time, and track intervention progress (Guralnick, 2000). However, practitioners have discouraged use of developmental age as a unitary construct to represent a child's potential across all domains because it does not reflect the high variability within and across domains among children with developmental disabilities (Greenspan \& Meisels, 1996). Developmental age from multiple-domain assessments such as the Bayley Scales of Infant Development (BSID; 2nd ed.; Bayley, 1993) or Battelle Developmental Inventory (BDI; 2nd ed.; Newborg, 2005) can be used to identify a variety of types of impairments in children with developmental disabilities (e.g., motor, sensory, or cognitive impairments) that can interact with language and influence success in communication intervention. Whereas multiple-domain developmental age assessments may be needed to identify domains of impairment and verify children for services, other strategies for assessing developmental and communicative potential should be considered for children with widely disparate skills across domains in order to assess and predict their skill development over time.

When some aspect of a child's developmental disability directly interferes with the child's ability to perform the standardized tasks within one domain, clinical guidelines often recommend substituting assessments from a closely related domain. For children with severe language impairments, particularly children who are not yet speaking, clinicians and researchers tend to rely on estimates of nonverbal cognition rather than direct expressive language measures (Hay \& Brieger, 2000) to characterize present developmental skills and estimate future cognitive and language potential. Researchers typically estimate nonverbal cognition for children under 2 years with either the Bayley or Battelle multiple-domain composite scores (Ulvund \& Smith, 1996) or with cognitive subtests of the same measures (Rose, Feldman, Jankowski, \& Van Rossem, 2005). Those cognitive age estimates are used as benchmarks against which to compare or predict language skill development and outcomes based on estimates of nonverbal cognitive abilities. For instance, nonverbal cognitive skills at 12 months predicted receptive and expressive language skills at 36 months for typically developing children (Rose, Feldman, \& Jankowski, 2009). Nonverbal cognitive scores also strongly predicted language skills for toddlers with delayed language (Oliver, Dale, \& Plomin, 2004) and toddlers di- agnosed with autism spectrum disorder (Luyster, Kadlec, Carter, \& Tager-Flusberg, 2008).

However, standardized nonverbal cognition measures may not accurately represent cognitive skill or language potential in young children with physical and expressive language impairments. Although verbal and nonverbal skills are moderately correlated in children with typical development (as would be expected for any skills with linear age-related expectations), levels and rates of verbal and nonverbal skill acquisition can differ substantially in children with developmental disabilities, particularly for sensorimotor tasks with high motor response demands (Dunst, 1998). Children with neurological and/or physical impairments frequently have co-occurring language, social, and/or cognitive deficits, as well as difficulty completing tasks of high motor complexity, such as those used in nonverbal cognitive assessment under 2 years. In general, domain subtests such as cognition on the Battelle and Bayley measures tend to be more accurate with children over 2 years; there are problems with discriminative validity for atrisk children under 2 years when used as a single estimate of a child's skills (Gerkin, Eliason, \& Arthur, 1994). Both instruments emphasize manipulation tasks to assess nonverbal skills in the cognitive subtests at 2 years and younger. For instance, these cognitive subtests rely on such motor behavior probes as "uncovers a hidden toy," "reaches around a barrier to obtain a toy," "transfers objects from hand to hand," "pulls string adaptively to secure a ring," and "picks up a cube."

Of the test items for children ages 9-24 months on the cognitive subtest of the BSID, children with limited hand or arm control would be physically unable to complete at least $70 \%$ of test items, regardless of their cognitive skills (Cress, 2002). A modified version of the BSID in which the motor and language components were eliminated had high internal consistency in young children with physical impairments with respect to the cognitive domains included in the original assessment (Guerette, Tefft, Furumasu, \& Moy, 1999). However, such a restricted estimate removes linguistic information that can be critical to targeted skill estimation and prediction for speech-language pathologists. We need a more reliable and representative strategy with which to estimate developmental or cognitive age if we wish to use that information to characterize or predict language skills in children with developmental disabilities.

Other closely related domains such as receptive language or expressive gesture may be more accurate and reliable sources for estimates of language and/or cognitive skills when a young child's physical disability interferes with his or her performance on standardized expressive language and nonverbal cognitive tasks. For infants with low birth weight and prematurity, language skills are generally less dependent on motor abil- 
ity than are other cognitive skills (Ulvund \& Smith, 1996). For adolescents and adults with cerebral palsy, Pueyo, Junque, Vendrell, Narberhaus, and Segarra (2008) found receptive vocabulary measures along with visuospatial abilities were the best predictors of cognitive performance on the Raven's Coloured Progressive Matrices test (Raven, Raven, \& Court, 2003). Many studies have also demonstrated the effectiveness of expressive gestures and/or rate of communication acts in children with disabilities to predict later receptive language skills (Bavin et al., 2008; Wetherby, Lonigan, Easterly, \& Stannard, 2002), expressive language skills (Iverson \& Goldin-Meadow, 2005), as well as both receptive and expressive language skills (Bates, Thal, Fenson, Whitesell, \& Oakes, 1989; Luyster et al., 2008). Observed gestures and communication rates were representative of expressive communication functioning in individuals with severe cognitive deficits for both intentional and nonsymbolic communicators (McLean, Brady, McLean, \& Behrens, 1999). Communication rate and level of gesture were significant predictors of language outcomes in preschool children with developmental disabilities (Brady, Marquis, Fleming, \& McLean, 2004).

Receptive language and expressive gesture can be assessed with minor modifications to a child's indicating response in ways that are potentially accessible to children with motor impairments; for instance, children may substitute whole-hand reaching for index finger pointing to indicate response to receptive language probes, or substitute idiosyncratic gestures (e.g., hand lift) for standard gestures (e.g., reach) on expressive gesture probes. Research has indicated that receptive language measures can provide a distinctly different estimate of skills that is less influenced by the motor impairments of children with physical impairments than nonverbal cognition measures. In toddlers with physical and/or neurological impairments, cognitive subscores on the BDI (Newborg, Stock, Wnek, Guidibaldi, \& Svinicki, 1984) were not significantly different from the children's developmental age estimates that incorporated low motor and adaptive skill domain scores, but receptive language estimates were consistently and reliably higher than either cognition or developmental age estimates at all ages tested (Ross \& Cress, 2006). If the cognitive subtests were used to characterize or predict language potential for these toddlers with physical impairments, the children's skills would be estimated as much as 6 months lower than when based on receptive language scores from the same developmental age measure.

Similarly, by relying on natural communicative gestures, rate of communication acts can be assessed reliably in children with severe motor impairments who produce recognizable intentional communication acts. Rate of communicative acts (how often a child commu- nicates using gestures, sounds, or spoken words within a standard play sample) can be measured through assessment tools such as the Communication and Symbolic Behavior Scales (CSBS; Wetherby \& Prizant, 1993), which uses action-based toys, books, and play materials to provide children with opportunities to initiate intentional communication acts. Intentional communication includes any adult-directed conventional or idiosyncratic gestures or the coordination of gestures and vocalizations used to convey messages to a partner in an interactive context (Wetherby \& Prizant, 1993). CSBS assessment procedures have been adapted successfully for children with physical impairments by making modifications to the type of probes used (Cress et al., 2000). For instance, small, manipulable objects may be substituted for Cheerios with children who cannot eat by mouth. Although normative data from the CSBS is available for children up to 36 months of age, age equivalents may be applied to children outside the norming range if a child's performance is not better than the average (median) child from the highest age range. Consequently, administration of the task trials is appropriate for obtaining descriptive information such as rate of communication for children older than 36 months. For children and adults with severe cognitive deficits (McLean et al., 1999) and toddlers with physical impairments (Cress et al., 2000), the reported rate of communicative acts was slower than expected for typically developing peers, but indicative of relative communication skills compared with other estimates of communication.

In summary, use of receptive language measures together with expressive gestural communication may estimate developmental age as effectively as traditional multiple-domain assessments. By reducing the motor requirements used in the assessment of developmental age in young children with disabilities, a communication-based estimation of developmental age may be justified for use with children whose developmental age composite scores may otherwise be limited by their physical skills. If there were empirical justification for substituting a communication composite for traditional multiple-domain assessments of developmental age, fewer children with physical and neurological impairments would be misidentified in the diagnosis and prediction of their cognitive and linguistic skills associated with developmental age assessments. Although supplementing developmental age assessment with other communication measures is a common clinical strategy, there has not been any research-based rationale for substituting these communication-based assessments as a specific estimate of developmental age in children with developmental disabilities.

In the present study, we use confirmatory factor analysis to compare assessments of developmental age from a typical multiple-domain approach (BDI) with a combi- 
nation of receptive language and expressive communication measures in young children with developmental disabilities who were at risk for long-term reliance on augmentative and alternative communication (AAC). Although it would have been possible to conduct a simpler analysis in which the communication-based assessments were initially averaged into a composite communication score, this would not have addressed the primary research question of this study because there would be no empirical basis underlying the theoretical belief of the communication composite as a measure of developmental age for children with developmental disabilities. The confirmatory latent factor analysis was thus necessary as an initial step to test whether a communication-based composite represents a reliable and unidimensional latent construct in the first place, and thus whether it is clinically justifiable to substitute communication-based measures to address some of the purposes for which developmental age assessments are typically used with children with developmental disabilities.

More specifically, we compared in this study two alternative assessment strategies for developmental age: use of a multiple-domain assessment strategy and use of a communication-based approach incorporating a combination of receptive language and expressive gesture measures. We addressed three research questions:

1. Does a communication-based assessment strategy reflect a coherent latent construct for estimating developmental age in young children with developmental disabilities who are at risk for long-term reliance on AAC?

2. Does a communication-based assessment of developmental age provide a rank ordering of children that is similar to the multiple-domain assessment strategy for estimating developmental age in young children with developmental disabilities at risk for longterm reliance on AAC?

3. Are there absolute differences in the age-equivalence scores as derived from the multiple-domain or communication-based assessments for this population?

\section{Method}

\section{Participants}

The sample included 42 children aged 9 months-27 months originating from an existing 50-participant longitudinal data set focused on communication development in children at risk for a long-term reliance on AAC secondary to neurological and/or physical etiologies (Cress, 1995). Participants were recruited from regional service agencies serving infants and toddlers with physical and/or neurological impairments. Only 42 of the 50 participants could be included in the present study be- cause eight children did not complete the full longitudinal sequence of the original study and, therefore, did not have usable data for the relevant measures examined in the present study. The children had a mean age of 18.2 months $(S D=3.95$, range $=9-26)$ at Time 1 , a mean age of 27.6 months $(S D=4.23$, range $=18-36)$ at Time 2 , and a mean age of 33.9 months $(S D=4.49$, range $=26-45)$ at Time 3. Children's chronological ages were corrected for number of weeks premature at all time points for children born before 37 weeks gestation.

All participants had developmental disabilities resulting from the following physical and/or neurological etiologies: cerebral palsy $(n=18)$, acquired brain injury/illness (e.g., meningitis, glutamic acidurea, traumatic brain injury; $n=11$ ), congenital developmental conditions (e.g., Opitz syndrome, achondroplasia, microcephaly; $n=6$ ), or congenital oral motor conditions (e.g., speech motor impairment, vocal fold paralysis; $n=$ 7). All the children also met criteria for being at risk for nonspeaking (i.e., long-term reliance on AAC), which included the presence of at least two of the following four characteristics: (a) birth anoxia, prematurity, or other prenatal factors; (b) feeding impairments or persistent oral-motor control problems; (c) delayed onset of vocalizations or speech relative to same-age peers; or (d) evidence of neuromotor deficits that have been associated with speech disorders (McDonald, 1980). Children could not yet be identified as nonspeaking long term because being nonspeaking is within typical limits for children 12-18 months of age.

Children were administered the BDI at three occasions during a longitudinal sampling term over an 18-month period. No modifications were made for the test administration beyond those published in the manual, and test-retest interval recommendations were followed. From administration of the BDI at the first occasion, the participants had a mean developmental age of 9.9 months (range $=2-21$ months), a mean receptive communication age of 14.2 months (range $=5-30.5$ months), and a mean expressive communication age of 10.2 months (range $=1-21.5$ months). All children demonstrated spoken expressive language skills at least $1 S D$ below the mean for their corrected ages and had been identified as having characteristics consistent with severe expressive speech impairments.

The families participating in the study were recruited from educational and clinical agencies in Nebraska, Kansas, and Iowa that provided services for children with physical and/or neurological impairments. Of the participants, $20 \%$ were from ethnic minority groups (7\% Hispanic, 5\% African American, 5\% reported "other" biracial status, 3\% Asian). Parental occupation and highest level of educational attainment were also obtained from participating families. Of primary wage earners in each family, 14 held an advanced academic degree (comprising a bachelor's or master's degree), 11 had some col- 
lege education, 16 obtained high school diplomas, and one did not complete high school. Three parents reported that they were the only parent in the household, and two children had grandparents who were their primary caregivers during data collection.

Parental occupations were evaluated using the International Socio-Economic Index of Occupational Status (ISEI) categories (Ganzeboom \& Treiman, 1996). The average occupational score was 45.05 (standardized midpoint $=40$ ). Nineteen parents had scores higher than 40, placing them in the categorical equivalent of entrepreneurs, professional positions, or supervisors. Nineteen parents were at or below 40, placing them in the categorical equivalent of skilled labor or farmer. Four were not reportable on the ISEI categories as they were principally homemakers or students.

\section{Procedure}

The data were derived from home-based standardized assessments in a longitudinal study of communication development in children with neurological and/or physical developmental disabilities at risk for a long-term reliance on AAC (Cress, 1995). The children and their parents received 2- to 3-hr visits in their homes during which a number of measures of cognitive and communicative development were administered. All assessments were administered by the third author, a licensed speech-language pathologist with 15 years of experience administering assessments to children with physical and neurological impairments. During an 18-month period, each family received six total visits, only three of which contained relevant measures for the present study. The second relevant visit (Time 2) occurred approximately 9 months after the first and the third (Time 3) occurred 6 months after the second. Each child was assessed at three separate times over the 18-month period for a total of 126 assessment occasions.

\section{Measures}

The MacArthur Communicative Development Inventory (CDI): Words and Gestures (Fenson et al., 1993) is a vocabulary checklist that examines vocabulary comprehension and production and is completed by a child's parent or caregiver. Response items include single words or short phrases organized categorically (e.g., sound effects and animal sounds, food and drink, body parts, etc.) and checklist columns for "understands" and "understands and says" organized by items such as the following in the "toys" category: ball, balloon, block, book, bubbles, doll, pen, and toy. Although the entire CDI was administered to participants, only the number of words understood was interpreted for this study.
The BDI is a standardized measure that assesses developmental age from a composite score consisting of the following domains: motor, cognitive, language (including both receptive and expressive), personal/social, and adaptive skills. Information for participant scores on individual items is obtained through direct observation, parental report, or examiner probes.

The Sequenced Inventory of Communication Development-Revised (SICD; Hedrick, Prather, \& Tobin, 1984) is a standardized measure used to assess receptive and expressive communication skills with young children. The SICD is based on a combination of parental report and/or behavioral observation in natural and prompted interactions.

The CSBS is a normed and standardized assessment of children's communicative and symbolic skills. Although the entire CSBS was administered addressing a variety of communicative domains, for the purpose of this study only the rate of communicative acts (i.e., gestures, verbalizations, and vocalizations) was used to allow a meaningful comparison with the raw data from the other assessments; the entire gesture cluster could not be included in the analysis because of the need to compare raw scores between measures rather than standardized composite scores. All children who produced intentional communication acts included some gestures and vocalizations, and some children had spoken words and sentences (verbalizations) or word approximations; children's signs, including symbolic idiosyncratic signs (e.g., a mouth/head gesture that represented "more") were counted as gestures but not spoken words for this assessment.

Administration of the CSBS involves setting up communicative temptations for young children (e.g., windup toy or jar with Cheerios in it) during which time the child observes the item in action (e.g., toy moving, jar opened, and a Cheerio given to the child). The child is then presented with the item and scored on his or her independent communicative attempts to get an adult to act on that item (e.g., give a wind up toy again or give another Cheerio), to comment on that item, or to interact socially. The CSBS: Normed edition counts all child communicative acts produced across multiple turns with nine temptations (including children's responses to communication breakdowns), allowing for a wide range of possible communication rates between children. For this study, children were administered the standard CSBS protocol by the third author, and videotaped CSBS interactions were coded using standard CSBS criteria for intentional communication acts. Research assistants who had completed a 2-month-long training period to become reliable at coding the complete CSBS: Normed edition scored the communication acts from video. The only adaptations to the CSBS administration were substituting some temptations (e.g., different objects than Cheerios in the jar as a temptation for children 
who were not oral feeders) and holding objects close to children's hands in request temptations for children who did not have independent grasp-and-release skills.

\section{Results}

\section{Analytic Strategy}

Means, standard deviations, and Pearson correlations among the original measures across all sampling occasions are given in Table 1; these means, standard deviations, and correlations are shown separately by sampling occasion for Time 1 (see Table 2), Time 2 (see Table 3), and Time 3 (see Table 4). All measures included in the multiple-domain and communication-based assessment strategies were significantly and similarly positively correlated with each other (correlations ranged from .6 to .9) at each time of observation as well as when compiled across observation periods.

Given that the observed measures were continuous indicators, we chose a confirmatory factor analysis (CFA) to test the dimensionality of these indicators, although we used robust maximum likelihood estimation within Mplus Version 6.0 (Muthén \& Muthén, 19982010) in all factor analyses given some observed skewness and kurtosis in the indicators. The indicators were transformed to $z$ scores so as to eliminate estimation problems due to widely different measurement scales. The models were identified by constraining each factor variance to 1 and each factor mean to 0 . Initially, we fit separate single-factor confirmatory models for each of the two versions of developmental age, the multiple-domain assessment strategy and communication-based assessment strategy, in order to examine the fit of the out- comes within each developmental age factor. We then estimated additional two-factor models to examine the correlation between the developmental age factors under each approach.

Because the indicators to be analyzed were collected longitudinally, responses from the same child are more likely to be related than responses from different children. To address this dependency, we used a clustered sampling correction via the CLUSTER option in Mplus 6.0 , in which the standard errors of the model parameters and the fit statistics of the model are corrected for the additional person-related dependency. This fixed-effect approach is commonly used when modeling clustered samples and is also appropriate for longitudinal samples, in which it can be used to account for the same type of person dependency. Results (as presented next) were largely similar with or without this clustering correction, however.

We used three indices to evaluate the quality of the fit in the CFA models: the obtained chi-square $\left(X^{2}\right)$, the comparative fit index $(\mathrm{CFI})$, and the root-mean-square error of approximation (RMSEA). The chi-square value is an index of the extent to which the observed variances and covariances are predicted by the system of equations specified in the model. A nonsignificant (small) chi-square is desirable (i.e., no significant discrepancy between the model and the actual data), and additional indices are also used to assess fit. The CFI is one such goodness-of-fit measure, where values above .90 or .95 indicate acceptable and excellent fit, respectively. The RMSEA is a measure of lack of fit, where values below .08 or .05 indicate acceptable or excellent fit, respectively. We examined local fit by using standardized residuals for the magnitude of the unexplained covariance

Table 1. Simple correlations among observed measures and descriptive statistics.

\begin{tabular}{|c|c|c|c|c|c|c|c|c|c|}
\hline Measure & 1 & 2 & 3 & 4 & 5 & 6 & 7 & 8 & 9 \\
\hline 1. Cognitive (BDI) & & & & & & & & & \\
\hline 2. Adaptive (BDI) & $.804^{*}$ & - & & & & & & & \\
\hline 3. Personal/Social (BDI) & $.888^{*}$ & $.800^{*}$ & - & & & & & & \\
\hline 4. Motor (BDI) & $.797^{\star}$ & $.843^{*}$ & $.764^{*}$ & - & & & & & \\
\hline 5. Receptive Language (BDI) & $.879 *$ & $.760 *$ & $.929 *$ & $.756^{*}$ & - & & & & \\
\hline 8. Receptive Vocabulary (CDI) & $.774^{*}$ & $.637^{*}$ & $.820^{*}$ & $.604^{*}$ & $.825^{\star}$ & $.751^{*}$ & $.598^{*}$ & - & \\
\hline 9. Receptive Language (SICD) & $.868^{*}$ & $.757^{*}$ & $.923^{*}$ & $.765^{\star}$ & $.918^{*}$ & $.872^{*}$ & $.759^{\star}$ & $.854^{*}$ & - \\
\hline M & 21.800 & 32.370 & 53.100 & 40.010 & 15.400 & 14.990 & 1.310 & 130.980 & 30.900 \\
\hline Variance & 57.335 & 159.947 & 579.895 & 567.011 & 27.668 & 64.803 & 3.137 & 15388.899 & 351.900 \\
\hline
\end{tabular}

BDI = Battelle Developmental Inventory; CSBS = Communication and Symbolic Behavior Scales; CDI = MacArthur Communicative

Development Inventory; SICD = Sequenced Inventory of Communication Development.

${ }^{*} p<.01$. 
Table 2. Simple Pearson correlations among observed measures and descriptive statistics for time 1 observations.

\begin{tabular}{|c|c|c|c|c|c|c|c|c|c|}
\hline Measure & 1 & 2 & 3 & 4 & 5 & 6 & 7 & 8 & 9 \\
\hline 1. Cognitive (BDI) & - & & & & & & & & \\
\hline 2. Adaptive (BDI) & $.666^{*}$ & - & & & & & & & \\
\hline 3. Personal/Social (BDI) & $.775^{*}$ & $.623^{*}$ & - & & & & & & \\
\hline 4. Motor (BDI) & $.695^{*}$ & $.692^{*}$ & $.667^{*}$ & - & & & & & \\
\hline 5. Receptive Language (BDI) & $.697^{*}$ & $.440^{*}$ & $.805^{*}$ & $.614^{*}$ & - & & & & \\
\hline 6. Expressive Language (BDI) & $.670^{*}$ & $.473^{*}$ & $.776^{*}$ & $.603^{*}$ & $.716^{*}$ & - & & & \\
\hline 7. Communicative Rate (CSBS) & $.592^{*}$ & $.510^{*}$ & $.744^{*}$ & $.688^{*}$ & $.709^{*}$ & $.582^{*}$ & - & & \\
\hline 8. Receptive Vocabulary (CDI) & $.516^{\star}$ & $.428^{*}$ & $.539^{*}$ & $.456^{\star}$ & $.580^{*}$ & $.602^{*}$ & $.396^{\star}$ & - & \\
\hline 9. Receptive Language (SICD) & $.726^{*}$ & $.609^{*}$ & $.832^{\star}$ & $.749^{\star}$ & $.807^{*}$ & $.729^{*}$ & $.686^{*}$ & $.683^{*}$ & 一 \\
\hline M & 17.950 & 26.220 & 39.920 & 31.600 & 12.270 & 10.600 & 0.800 & 65.470 & 21.490 \\
\hline Variance & 27.228 & 88.846 & 244.943 & 344.041 & 12.769 & 25.169 & 1.398 & 8607.229 & 138.414 \\
\hline
\end{tabular}

${ }^{*} p<.01$.

between indicators (i.e., as available via the RESIDUAL option within Mplus), and we evaluated practical significance by examining the magnitude of the standardized factor loadings. For a more complete description of procedures for CFA model evaluation, see Brown (2006).

\section{Multiple-Domain Developmental Age}

Initially, fit of the six-indicator model from the BDI, including cognitive, adaptive, personal/social, motor, receptive language, and expressive language, was acceptable only according to the CFI, $x^{2}(9, N=42)=39.68$, $p<.01, \mathrm{CFI}=0.95$, RMSEA $=0.16$, suggesting that some indicators within this domain were either more or less related to one another beyond their expected relation due to their common latent factor of developmental age. Examination of the residuals for covariances (i.e., the difference between the model-predicted and observed indicator covariances) indicated that the adaptive and motor subtests were more related than the model predicted. Adding an additional relationship between the residual variances of these subtests had theoretical rationale for this population given that motor ability impacts a child's capacity for independently carrying out activities of daily living.

The receptive subtest of the BDI was a theoretical portion of each subscale. In the two-factor model, examined next, however, the factor loadings from this indicator could only be used for one of the two factors given our interest in examining the correlation between them. Because the communication-based model was a focus of our research hypotheses, the inclusion of all relevant receptive language indicators was necessary. We chose to keep the receptive subtest in the communication-based subscale and, consequently, reanalyzed the multiple-domain developmental age model without the

Table 3. Simple Pearson correlations among observed measures and descriptive statistics for Time 2 observations.

\begin{tabular}{|c|c|c|c|c|c|c|c|c|c|}
\hline Measure & 1 & 2 & 3 & 4 & 5 & 6 & 7 & 8 & 9 \\
\hline 1. Cognitive (BDI) & - & & & & & & & & \\
\hline 2. Adaptive (BDI) & $.750^{*}$ & - & & & & & & & \\
\hline 3. Personal/Social (BDI) & $.826^{*}$ & $.741^{*}$ & - & & & & & & \\
\hline 4. Motor (BDI) & $.779^{*}$ & $.851^{*}$ & $.748^{*}$ & - & & & & & \\
\hline 5. Receptive Language (BDI) & $.849^{*}$ & $.757^{*}$ & $.885^{*}$ & $.786^{*}$ & - & & & & \\
\hline 6. Expressive Language (BDI) & $.900^{*}$ & $.794^{*}$ & $.886^{*}$ & $.785^{*}$ & $.826^{*}$ & - & & & \\
\hline 7. Communicative Rate (CSBS) & $.684^{*}$ & $.657^{*}$ & $.748^{*}$ & $.705^{*}$ & $.798^{*}$ & $.702^{*}$ & - & & \\
\hline 8. Receptive Vocabulary (CDI) & $.755^{*}$ & $.561^{*}$ & $.865^{*}$ & $.591^{*}$ & $.831^{*}$ & $.774^{*}$ & $.688^{*}$ & - & \\
\hline 9. Receptive Language (SICD) & $.870^{*}$ & $.692^{*}$ & $.946^{*}$ & $.726^{*}$ & $.908^{*}$ & $.875^{*}$ & $.792^{*}$ & $.884^{*}$ & - \\
\hline M & 21.950 & 32.070 & 52.640 & 39.100 & 15.790 & 15.260 & 0.186 & 19.305 & 2.798 \\
\hline Variance & 49.656 & 130.068 & 412.186 & 523.405 & 21.831 & 56.979 & 1.457 & 15278.298 & 320.890 \\
\hline
\end{tabular}

${ }^{*} p<.01$. 
Table 4. Simple Pearson correlations among observed measures and descriptive statistics for Time 3 observations.

\begin{tabular}{lccccccccc}
\hline Measure & 1 & 2 & 3 & 4 & 5 & 6 & 7 & 8 & 9 \\
\hline 1. Cognitive (BDI) & - & & & & & & & & \\
2. Adaptive (BDI) & $.809^{*}$ & - & & & & & & & \\
3. Personal/Social (BDI) & $.926^{*}$ & $.814^{*}$ & - & & & & & & \\
4. Motor (BDI) & $.798^{*}$ & $.875^{*}$ & $.768^{*}$ & - & & & & & \\
5. Receptive Language (BDI) & $.916^{*}$ & $.775^{*}$ & $.969^{*}$ & $.748^{*}$ & - & & & & \\
6. Expressive Language (BDI) & $.885^{*}$ & $.831^{*}$ & $.924^{*}$ & $.813^{*}$ & $.880^{*}$ & - & & & \\
7. Communicative Rate (CSBS) & $.647^{*}$ & $.636^{*}$ & $.726^{*}$ & $.751^{*}$ & $.726^{*}$ & $.708^{*}$ & - & & \\
8. Receptive Vocabulary (CDI) & $.351^{*}$ & $.451^{*}$ & $.560^{*}$ & $.445^{*}$ & $.512^{*}$ & $.500^{*}$ & $.395^{*}$ & - & \\
9. Receptive Language (SICD) & $.892^{*}$ & $.754^{*}$ & $.954^{*}$ & $.771^{*}$ & $.934^{*}$ & $.886^{*}$ & $.776^{*}$ & $.576^{*}$ & - \\
$M$ & 24.870 & 38.130 & 65.750 & 47.050 & 17.950 & 18.720 & 1.900 & 177.490 & 39.180 \\
Variance & 73.394 & 198.574 & 817.782 & 683.741 & 33.587 & 80.256 & 6.069 & 16848.941 & 469.204 \\
\hline
\end{tabular}

${ }^{*} p<.01$.

receptive subtest. The resulting five-indicator model fit was acceptable except for the RMSEA (which tends to favor more parsimonious models with larger remaining degrees of freedom; see Brown, 2006), $x^{2}(5, N=42)$ $=20.35, p=.001, \mathrm{CFI}=0.97$, RMSEA $=0.16$. As with the six-indicator model, examination of the residuals for the model-predicted covariances indicated that the adaptive and motor subtests within the five-indicator model were more related than the single-factor model predicted.

After accounting for this additional residual relationship, the modified measurement five-indicator model was acceptable by all indices, $\chi^{2}(4, N=42)=3.13, p=$ $.54, \mathrm{CFI}=1.00$, RMSEA $=0.00$. The range of standardized loadings of each indicator to the factor was 0.830.95 , indicating very strong correlations with the latent factor. The modified five-indicator developmental age factor model fit significantly better than the original model, $\chi^{2}(1, N=42)$ difference $=12.03, p \leq .01$. Modelbased reliability (omega), the overall proportion of variance in the indicators due to the latent factor, was 0.92, as derived from the squared sum of the factor loadings relative to that plus the sum of the residual variances and twice any residual covariances (Brown, 2006). In summary, the multiple-domain developmental age factor had excellent model fit and reliability as well as a majority of its variance explained by the latent factor in each indicator ( $R^{2}$ ranging from .69 to .90$)$, indicating a coherent unidimensional construct.

\section{Communication-Based Developmental Age}

Overall, the fit of the four-indicator model for the communication-based approach was acceptable except for the RMSEA (as expected for a model with relatively few degrees of freedom), $\chi^{2}(2, N=42)=8.87, p=$ $.01, \mathrm{CFI}=0.95, \mathrm{RMSEA}=0.17$. Omega model-based re- liability was 0.94 , and the range of standardized loadings was .77 (rate of communicative acts from the CSBS) to .98 (receptive language portion of the SICD). Thus, the communication-based factor of developmental age also appeared to have good model fit and reliability as well as a majority of its variance explained by the latent factor in each indicator ( $R^{2}$ ranging from .60 to .95), also indicating a coherent unidimensional construct as hypothesized.

\section{Comparison of the Two Developmental Age Factors}

Overall, fit for the nine-indicator model of the two latent developmental age factors (see Figure 1) was acceptable by all indices, $X^{2}(25, N=42)=45.40, p=.01$, CFI $=0.98$, RMSEA $=0.08$. Table 5 provides the estimated model parameters. The multiple-domain and communication-based developmental age latent factors were correlated at $r=.98$, indicating they were functionally equivalent with respect to the rank order of individual differences in developmental age. Reliability in the twofactor model solution was slightly higher for the communication-based developmental age factor (.94) than for the multiple-domain developmental age factor (.91). Figure 2 depicts the factor score distribution of each latent factor as obtained via empirical Bayes predictions for each case in the sample. As shown in the bottom panel of Figure 2, there is excellent correspondence between the predicted factor scores along the entire trait dimension, further supporting their functional equivalence. Finally, each set of predicted factor scores showed excellent factor determinacy, or correlation with the model-based latent trait (.99 for each). The factor score standard errors were .141 and .146 for the multiple-domain and communication-based factors, respectively, 


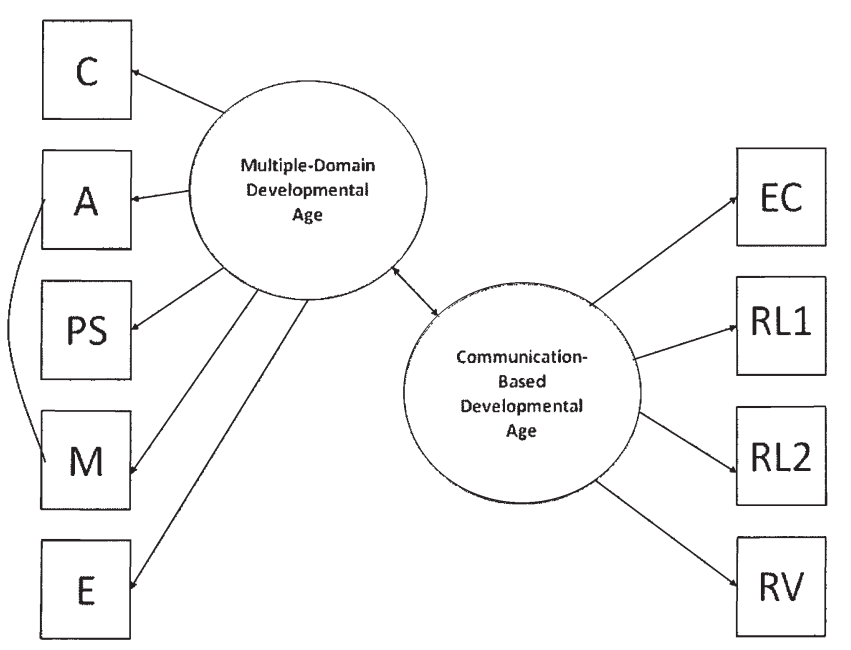

Figure 1. The multiple-domain latent factor was composed of the Battelle Developmental Inventory (BDI) subtests: Cognitive (C), Adaptive (A), Personal/Social (PS), Motor (M), and Expressive Language (E). The communication-based latent factor was composed of several language and communication measures: rate of communication acts from the Communication and Symbolic Behavior Scales (CSBS) as a measure of expressive communication (EC), the Receptive Language subtest of the BDI (RL1), the Receptive Language portion of the Sequenced Inventory of Communication Development (SICD; RL2), and the Receptive Vocabulary measure from the MacArthur Communicative Development Inventories (CDI; RV). Residual variances were also estimated for each indicator, as was a single covariate between the residuals for the $A$ and $M$ subtests.

indicating similar levels of precision in considering the most likely factor score for each person, in addition to comparable levels of model fit and reliability.

\section{Comparison of the Two Developmental Age- Equivalence Scores}

The preceding factor analyses were necessary to demonstrate that the multiple-domain approach and the communication-based approach each forms a coherent unidimensional factor, such that a single trait is being measured by each, which is an important precursor to considering the summaries of developmental age that could be provided by these two sets of measures. But because latent factors do not have inherent scales, a latent factor score does not have a real-world counterpart that indicates a given child's ability. Thus, although the near-perfect correlation between the two developmental age factors indicates that they rank order individuals in the same way, the latent factor models cannot directly address the primary issue at stake in children with disabilities - whether developmental age would be assessed as significantly higher in the communicationbased approach than in the traditional multiple-domain approach. To address this issue, we attempted to cal-
Table 5. Two-factor confirmatory factor model parameters.

\begin{tabular}{|c|c|c|c|}
\hline Item factor loadings & Estimate & SE & $\begin{array}{r}\text { Std. } \\
\text { estimate }\end{array}$ \\
\hline \multicolumn{4}{|c|}{ Multiple-domain developmental age } \\
\hline Cognitive (BDI) & 0.918 & 0.142 & 0.922 \\
\hline Adaptive (BDI) & 0.821 & 0.123 & 0.825 \\
\hline Personal/Social (BDI) & 0.972 & 0.074 & 0.976 \\
\hline Motor (BDI) & 0.805 & 0.109 & 0.808 \\
\hline Expressive Language (BDI) & 0.925 & 0.091 & 0.929 \\
\hline \multicolumn{4}{|c|}{ Communication-based developmental age } \\
\hline Receptive Language (BDI) & 0.952 & 0.092 & 0.956 \\
\hline Communicative Rate (CSBS) & 0.772 & 0.123 & 0.774 \\
\hline Receptive Vocabulary (CDI) & 0.926 & 0.079 & 0.879 \\
\hline Receptive Language (SICD) & 0.964 & 0.077 & 0.968 \\
\hline $\begin{array}{l}\text { Multiple-domain and } \\
\text { communication-based } \\
\text { developmental age factor } \\
\text { covariance }\end{array}$ & 0.984 & 0.007 & 0.984 \\
\hline \multicolumn{4}{|l|}{ Residual variances } \\
\hline Cognitive (BDI) & 0.149 & 0.031 & 0.151 \\
\hline Adaptive (BDI) & 0.318 & 0.059 & 0.320 \\
\hline Personal/Social (BDI) & 0.047 & 0.012 & 0.048 \\
\hline Motor (BDI) & & 0.062 & 0.347 \\
\hline Receptive Language (BDI) & 0.086 & 0.014 & 0.086 \\
\hline Expressive Language (BDI) & 0.137 & 0.024 & 0.138 \\
\hline Communicative Rate (CSBS) & 0.399 & 0.119 & 0.401 \\
\hline Receptive Vocabulary (CDI) & 0.252 & 0.126 & 0.277 \\
\hline Receptive Language (SICD) & 0.062 & 0.020 & 0.063 \\
\hline $\begin{array}{l}\text { Adaptive and motor residual } \\
\text { covariance }\end{array}$ & 0.175 & 0.050 & 0.530 \\
\hline
\end{tabular}

Std. = standard.

culate age-equivalence scores for each of the nine indicators used in the latent factor models as described by their respective test manuals. However, for the communication-based factor, the CSBS and CDI did not have age-equivalence scores for children with very low or very high scores. Therefore, we used only age-equivalence scores for the BDI and the SICD to represent the communication-based factor, whereas age-equivalence scores were available for all five indicators to represent the multiple-domain factor at all ages sampled. The correlation between the five-indicator multiple-domain factor and the two-indicator communication-based factor (with just the BDI and SICD) remained at $r=.98$, indicating that the removal of the CSBS and CDI did not compromise the correspondence between the two factors for assessing developmental age.

We then conducted a variance components analysis in order to assess (for each factor) the intraclass correlation expressing the amount of variation between occasions and children relative to the amount of variation across the age-equivalence scores within the same occasion. We then estimated a model in which the fixed intercept and all variance components were then estimated separately per factor (i.e., a heterogeneous variance model) by using restricted maximum likelihood in 

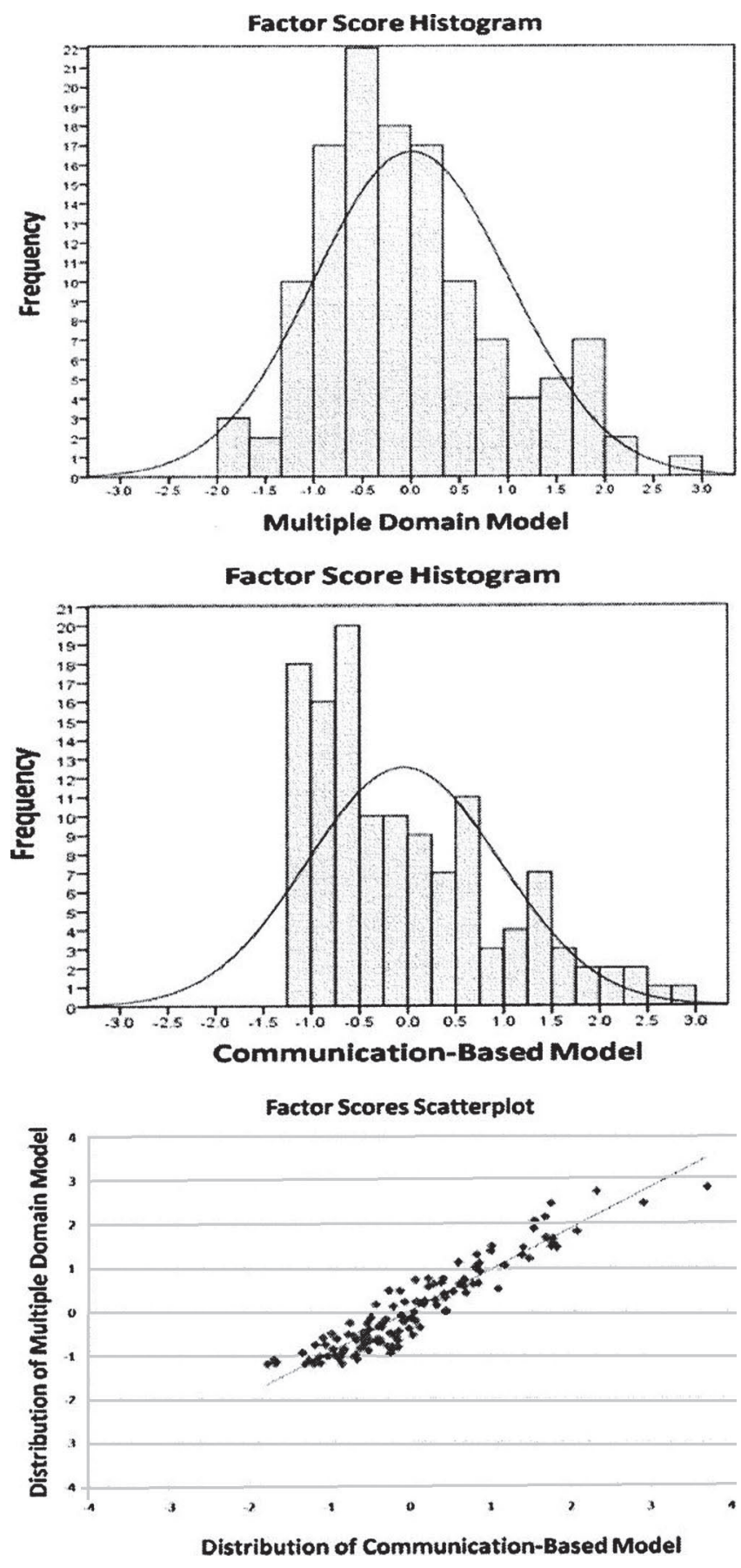

Figure 2. Multiple-domain and communication-based models: Factor score distribution comparisons.

SAS PROC MIXED (v. 9.2). For the multiple-domain approach, an intraclass correlation of 0.78 was found, indicating that $78 \%$ of the total variance across age-equivalence scores was systematic to the occasion and the individual and that $22 \%$ of the variance was due to unreliability, or differences between the five age-equivalence indicators at the same occasion for the same child (i.e., a reliability of 0.78 ). The intraclass correlation for the communication-based assessment strategy was 0.81 , indicating $19 \%$ of the variance was due to unreliability across the two age-equivalency indicators. Thus, comparable levels of reliability were achieved using the ageequivalence scores from either assessment strategy.

We then examined the potential differences between the two assessment strategies in the absolute estimate of developmental age within the same type of model, in which the separate indicators are essentially unitweighted to create an average developmental age for each strategy of assessment, but in which the differences between occasions were modeled at fixed effects. As hypothesized, children were evaluated as significantly lower in developmental age using the multiple-domain assessment strategy than when using the communication-based assessment strategy. Specifically, there was a significant main effect of assessment, $F(1,82)=13.9, p$ $=.0004$, such that the mean developmental age was significantly higher for the communication-based indicators $(M=18.4, S E=1.00)$ than for the multiple-domain indicators $(M=13.1, S E=0.98)$. There was also a significant main effect of occasion, $F(2,414)=225.6, p<.0001$, such that developmental age scores (averaged across the methods of assessment) increased from 11.6 at Time 1 to 16.3 at Time 2 to 19.3 at Time 3. However, we found no significant interaction between strategy and occasion, $F(2,414)=2.46, p=.087$, indicating that the advantages in the developmental age scores of 4.3, 5.8, and 5.6 for the communication-based indicators at Times 1, 2, and 3, respectively, were equivalent. See Figure 3 for a graphic representation of the developmental age estimates from the two assessment strategies at the three occasions.

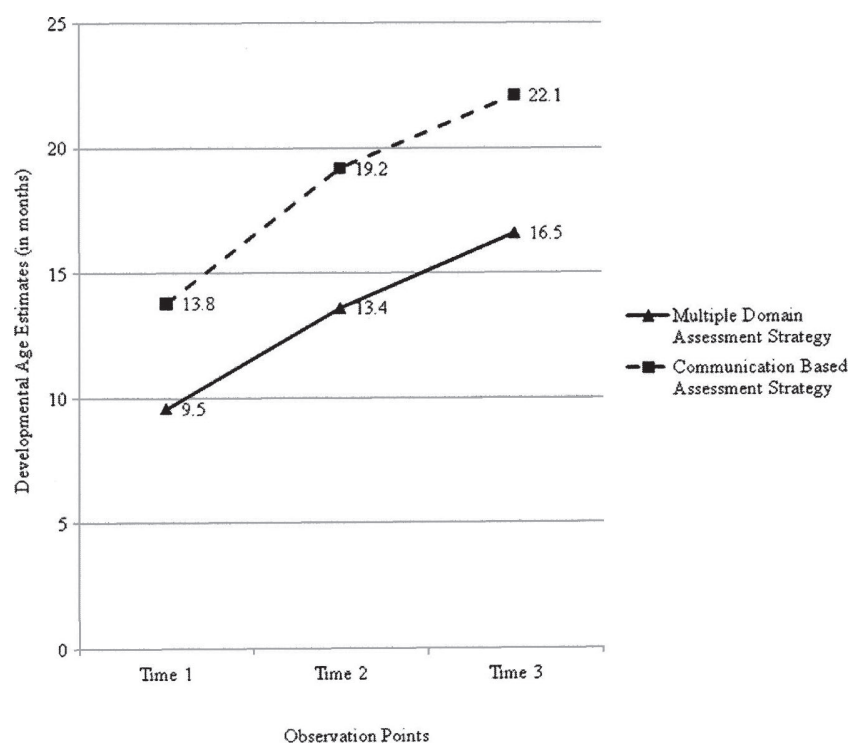

Figure 3. Multiple-domain and communication-based assessment strategies: Developmental age estimate comparisons. 


\section{Discussion}

In the present study, we examined the correspondence of two methods of assessing developmental age in young children at risk for long-term reliance on AAC. The first research question asked whether a communication-based assessment strategy including measures of receptive language and communicative acts formed a coherent and reliable unidimensional latent construct for estimating developmental age in these children. The answer is yes: The model was judged to be of acceptable fit for the data because of the strength of the goodness-of-fit measures $\left(x^{2}, C F I\right)$, and the latent factor had high reliability. Although the RMSEA measure was not within benchmark levels of acceptance, this is not surprising, considering that this statistic favors models with higher degrees of freedom reflecting greater parsimony, which would penalize four-indicator models such as ours that only have two remaining degrees of freedom. Furthermore, research suggests that with relatively small sample sizes, RMSEA is less of a concern when other fit indices strongly suggest "good" model fit (Brown, 2006). Each indicator used in the model had a statistically significant and meaningfully large standardized factor loading such that the majority of its variance was predicted by the developmental age latent factor. Thus, the combination of the receptive language subtests from the BDI and the SICD, the receptive vocabulary index from the CDI, and the rate of communicative acts obtained from the CSBS was an effective, coherent, and reliable strategy for the estimation of developmental age in this sample.

This analysis of the communication-based factor supports the use of a combination of receptive language and communication measures to reliably estimate the developmental age of children at risk for long-term reliance on AAC. This finding is consistent with the results of Pueyo et al. (2008) and Ross and Cress (2006), in which early measures of receptive language skills were found to be reliable developmental indicators for individuals with severe disabilities. In addition, these results are consistent with findings indicating that early communication skills could predict later cognitive and language skills in premature children (Ulvund \& Smith, 1996), typically developing toddlers (Wetherby et al., 2002), and young children with developmental disabilities (Brady et al., 2004).

The second research question asked whether the communication-based assessment strategy provided a similar rank ordering to the multiple-domain assessment strategy in estimating developmental age for this population. The answer is, again, yes: The present analyses demonstrated that the two latent factors for multiple-domain and communication-based developmental age were almost perfectly correlated, with similar factor score distributions and similar levels of reliability, indicating that they were functionally representing the same latent trait of developmental age. Overall, a combined receptive language and communication index was comparable to a multiple-domain assessment strategy and may be used to effectively substitute other strategies of estimating developmental age for young children with developmental disabilities.

Finally, the third research question asked whether scores derived from the multiple-domain and communication-based assessments differed significantly in their absolute estimates of age equivalence for developmental age. The answer is, again, yes: The ageequivalence scores derived from the communicationbased assessment were significantly higher than those derived from the multiple-domain strategy by an average of 5.3 months across all ages sampled. At Time 1, for instance, there was an average 18.4-months developmental age estimate for the communication-based strategy, versus an average 13.1-months developmental age estimate with the multiple-domain strategy. There was no significant difference in the relative discrepancy between the receptive language estimate and the multiple-domain estimate of developmental age across occasions of assessment. Children at all three sampling sessions (across average ages of 18.233.9 months) demonstrated a relative advantage of approximately 5 months for the receptive language strategy over the multiple-domain strategy in estimating developmental age. Even though the communicationbased age-equivalence score could only be constructed from the two receptive language measures for which age equivalence could be obtained at all ages for all children, there was still equivalent reliability between the communication indicators and the multiple-domain indicators. Therefore, not only was the communication-based assessment strategy able to reliably estimate the latent construct of developmental age, this strategy that avoided factors with potential motor confounds also resulted in a more favorable (and likely more valid) estimation of relative developmental age than the traditional multiple-domain strategy.

The significant discrepancy was expected and supports previous research that indicated multiple-domain assessments underestimated developmental age estimates for this population because of the physical load of manipulation tasks involved (Cress, 2002; Granlund, Olsson, \& Karlan, 1991). Ross and Cress (2006) found that BDI receptive language subtests were consistently higher than either the nonverbal cognition or the overall developmental age composite BDI scores for these children with physical impairments at all ages sampled. 
The implications of using traditional multiple-domain measures, therefore, would be lower expectations for developmental age than are warranted if considering the types of communication and language skills typically addressed in speech-language service delivery. The pattern of higher scores with the communication-based strategy than the multiple-domain strategy was consistent across all of the age groups of children with physical and developmental disabilities addressed in this study, from late infancy through preschool ages. If the purpose of administering a developmental age assessment was to characterize a child's overall developmental status or potential, for research or comparative purposes, then administering a traditional multiple-domain assessment would consistently underestimate developmental skills and potential for children with developmental disabilities at risk for long-term reliance on AAC. By relying on communication-based factors such as receptive language skills that are known predictors of later developmental skills (Chait \& Roy, 2008; Oliver et al., 2004), resulting estimates of developmental age from the communication-based model would be justified both by clinical relevance and consistency with previous research for characterizing and predicting language and cognitive skills of children with disabilities (Brady et al., 2004; McLean et al., 1999; Pueyo et al., 2008; Ross \& Cress, 2006).

Therefore, a communication-based assessment strategy is justified for estimating developmental age in children with developmental disabilities, particularly in children with known physical impairments that can influence their performance on multiple-domain developmental age assessments, when the predictor of interest is related to cognition or language skills. A multiple-domain assessment strategy may be necessary if the purpose of assessing developmental age is to verify for services and characterize a child's limitations across domains such as fine motor, adaptive, or traditional nonverbal cognitive skills. However, the communicationbased assessment strategies would be a more clinically justifiable strategy to estimate developmental age in order to predict language or cognitive potential, characterize current strengths and challenges, and plan interventions. By demonstrating a research basis for substituting communication-based assessments for more traditional assessments of developmental age, this study justifies alternative assessment strategies to estimate developmental age for children with physical or neurological impairments that are less likely to underestimate language and cognitive skills and misidentify children for diagnosis and prediction of communication skills in the assessment and intervention process.

\section{Limitations and Future Directions}

Several factors could potentially limit the populations to whom these results may be applicable. First, the sample size of 42 participants was small, and repeated measures across three occasions were used to obtain an adequate number of cases for analysis. Although appropriate statistical corrections were used to account for this dependency, the assumption of measurement invariance across age (i.e., equivalent measurement model parameters across waves, here) was made in estimating these models. It is important to recognize, however, that this same assumption is routinely made when using these instruments to assess children of different ages in research and practice more broadly.

CFAs generally require a large sample size (Brown, 2006), and the total sample size of 126 observations was on the low end of acceptability for CFA modeling. It is encouraging, however, that the latent factors showed strong cohesion in even a relatively small sample. Nevertheless, a larger number of children within this population would provide a more robust sample from which to replicate these CFA results. In general, however, children at risk for long-term reliance on AAC represent a small population that is often difficult to recruit. Any research conducted for this population of young children needs to creatively solicit sufficient group members. For example, in order to obtain a sufficient number of participants for this data set, participants were actively recruited within a three-state area for several years.

Another limitation for the generalization of these results is the heterogeneity of the population sampled. The participants had a variety of impairments and a wide range of skill levels and were all identified as having sufficient risk for not developing adequate verbal communication skills to meet all their communicative needs. Although a more homogenous group would be optimal, heterogeneity is a hallmark of this population. Other researchers addressing predictions in similar populations with developmental disabilities have reflected equivalent or greater variability in etiologies or skill ranges (Brady et al., 2004; McCathren, Yoder, \& Warren, 1999; Yoder, Warren, \& McCathren, 1998). Generalization of these results to individual children should reflect the high degree of variability that is expected for young children with severe disabilities. Although the pattern of higher receptive language estimates than overall developmental estimates was remarkably consistent for this population across all ages sampled, the extent of variability in this population would suggest the possibility of individual outliers in which receptive language scores were lower or equivalent to developmental age scores. Also, the data analyzed for this study were collected beginning in 1995 and analyzed retrospectively after the conclusion of the study. Since that time, many of the standardized assessments used in this study have been updated and revised (e.g., BDI; Newborg, 2005; CDI; Fenson et al., 2006). Even though new test editions tend to be similar to previous versions in terms of reliability and validity, it would be an appropriate expan- 
sion of the present research to replicate this study using updated assessment materials.

Additional research directions could be considered as further extensions to this study. An application of the communication-based model to additional populations of young children with disabilities with different ages and etiologies, including those with more specific motor impairments (e.g., cerebral palsy) or more general language problems (e.g., young children classified as "late talkers"), would broaden the populations for which this model has research-based application. Also, these results were tested for young children up to age 3, and it would be valuable to test whether the distinctions in the age-equivalence scores between the communication-based and multiple-domain assessment strategies would be demonstrated in older children with physical impairments. Although a negative link has been indicated between motor abilities and standardized cognitive measures, a detailed item analysis of the standardized cognitive assessments could be conducted in order to determine the precise questions from the standardized cognitive measures that are more closely associated with physical demands.

\section{Clinical Significance}

The consequences of using a communication-based assessment strategy have clear clinical implications. The present study provides research-based support for clinicians working with young children with physical or neurological disabilities (or who are suspected of having physical or neurological disabilities) to make use of a communication-based assessment strategy for estimating developmental age rather than the more commonly used multiple-domain assessment strategy, given their demonstrated functional equivalence in rank ordering individuals, but significantly higher absolute ability when assessed using measures of communication. For research purposes of estimating developmental age, administering the three receptive language subtests (BDI Receptive, SICD Receptive, and MacArthur CDI Words Understood) and one expressive communication subtest (CSBS Rate of Communicative Acts) would be appropriate and feasible ways to characterize developmental potential in this highly variable population.

For clinical application, in which multiple correlated assessments are less likely to be administered, the results of this study support the use of a composite of the shorter standardized receptive language subtests (the BDI or SICD) as a clinical stand-in for the complete communication-based model that would still represent higher but equally appropriate estimates of developmental skills and potential of children at risk for longterm reliance on AAC than the typical multiple-domain strategy. Because each of the four communication and receptive language measures used in the communica- tion-based model provides nuanced perspectives on developmental skills that may differ somewhat within individual children, it may be clinically useful to report the range of individual scores across each of the measures administered to more richly characterize a child's skills and developmental potential, in addition to averaging composite scores for the specific measures for developmental age estimation. A receptive language age rather than traditional developmental age criterion may also be considered as an alternative for administrative purposes when reviewing verification guidelines and clinical policies for children with developmental disabilities. By revising procedures and standards for diagnosis, verification, or prediction of communication skills on the basis of global multiple-domain developmental age estimates to use communication-based estimates instead, it is likely that fewer children with physical or neurological impairments will be underestimated for their cognitive and/or language skills based on a formal quantitative assessment of developmental skills. Further informal and family-based assessment is essential to accurately characterize communication skills in children with developmental disabilities and to account for variability in vocal, nonvocal, and augmented methods of conveying communication skills.

This study offers a communication-based alternative to professionals working with this young population that may be useful for assessing current skills as well as intervention planning. Although a multiple-domain assessment strategy is useful for obtaining sources of relative disability across domains, a communicationbased assessment strategy is practical for estimating language or other developmental potential for a child with impairments in motor or adaptive skills. Developmental age should not be assessed in a restrictive sense for determining a child's therapeutic potential. Every child should have access to treatment options that are not restricted by narrow decontextualized measures that attempt to characterize their skills in a single composite score such as developmental age. A complete communication assessment for children with developmental disabilities should include partner and environmental contributions to children's communication needs and strategies as well as open-ended information on children's multiple modes, functions, modifications, and purposes for expressing and understanding communicative messages.

In conclusion, a communication-based assessment strategy composed of a combination of the receptive language subtests from the BDI and the SICD, the receptive vocabulary index from the CDI, and the rate of communicative acts obtained from the CSBS is a useful and justifiable method for estimating developmental age in young children with developmental disabilities. A latent factor of developmental age indicated by these communication-based measures was reliable and 
highly correlated with a latent factor of developmental age indicated by a traditional multiple-domain assessment strategy, the BDI subscores. The equivalently reliable communication-based assessment strategy, however, resulted in significantly higher developmental age estimates by over 5 months relative to the traditional multiple-domain strategy. Establishing an alternative approach to estimating developmental age through receptive language and communication measures is clinically justifiable and important to minimize the potential limiting effect that fine and gross motor ability may have on the multiple-domain developmental age scores for children with physical impairments. Clinicians working with young children with developmental or physical disabilities and who are at risk for long-term reliance on AAC should consider using a communication-based approach as an alternative to the multipledomain approach for characterizing a child's current developmental skills, for identifying a child's strengths and challenges, as well as for planning interventions.

Acknowledgments - This research was supported in part by the National Institute on Deafness and Other Communication Disorders Grant K08 DC00102-01A1, awarded to the third author. Portions of this research were presented at the November 2010 annual convention of the American Speech-LanguageHearing Association in Philadelphia, PA. We also appreciate the contributions of the children and families who participated in this research.

\section{References}

Bates, E., Thal, D., Fenson, L., Whitesell, K., \& Oakes, L. (1989). Integrating language and gesture in infancy. Developmental Psychology, 25, 1004-1019.

Bavin, E. L., Prior, M., Reilly, S., Bretherton, L., Williams, J., Eadie, P., \& ... Ukoumunne, O. C. (2008). The Early Language in Victoria Study: Predicting vocabulary at age one and two years from gesture and object use. Journal of Child Language, 35, 687-701.

Bayley, N. (Ed.). (1993). Bayley Scales of Infant DevelopmentSecond edition: Manual. San Antonio, TX: The Psychological Corporation.

Brady, N. C., Marquis, J., Fleming, K., \& McLean, L. (2004). Prelinguistic predictors of language growth in children with developmental disabilities. Journal of Speech, Language, and Hearing Research, 47, 663-677. doi:10.1044/1092-4388(2004/051).

Brown, T. A. (Ed.). (2006). Confirmatory factor analysis for applied research. New York, NY: Guilford.

Chait, S., \& Roy, P. (2008). Early phonological and sociocognitive skills as predictors of later language and social communication outcomes. Child Psychology and Psychiatry, 49, 635-645.
Cress, C. (1995). Communicative and symbolic precursors to AAC development. Unpublished NIH grant document (NIDCD), University of Nebraska-Lincoln.

Cress, C. (2002). Expanding children's early augmented behaviors to support symbolic development. In J. Reichle, D. Beukelman, \& J. Light (eds.), Implementing an augmentative communication system: Exemplary strategies for beginning communicators (pp. 219-272). Baltimore, MD: Brookes.

Cress, C., Shapley, K., Linke, M., Havelka, S., Dietrich, C., Elliot, J., \& Clark, J. (2000, August). Characteristics of intentional communication in young children with physical impairments. Poster presented at the International Society for Augmentative and Alternative Communication Conference, Washington, DC.

Dunst, C. J. (1998). Sensorimotor development and developmental disabilities. In J. A. Burack, R. M. Hodapp, \& E. Zigler (eds.), Handbook of mental retardation and development (pp. 135-182). New York, NY: Cambridge University Press.

Fenson, L., Dale, P. S., Reznick, J. S., Thal, D., Bates, E., Hartung, J. P., \& ... Reilly, J. S. (eds.). (1993). MacArthur Communicative Development Inventories. San Diego, CA: Singular.

Fenson, L., Marchman, V. A., Thal, D. J., Dale, P. S., Reznick, J. S., \& Bates, E. (eds.). (2006). MacArthur-Bates Communicative Development Inventories (2nd ed.). Baltimore, MD: Brookes.

Ganzeboom, H., \& Treiman, D. (1996). Internationally comparable measures of occupational status for the 1988 International Standard Classification of Occupations. Social Science Research, 25, 201-239.

Gerkin, K. C., Eliason, M. J., \& Arthur, C. A. (1994). The assessment of at-risk infants and toddlers with the Bayley Mental Scale and the Battelle Developmental Inventory: Beyond the data. Psychology in the Schools, 31, 181-187.

Granlund, M., Olsson, C., \& Karlan, G. R. (1991). Investigating the relationships among motor ability, cognitive ability, and communication of persons with profound mental retardation. Scandinavian Journal of Educational Research, 35, 31-55.

Greenspan, S. I., \& Meisels, S. J. (1996). Toward a new vision for the developmental assessment of young children. In S. J. Meisels \& E. Fenichel (eds.), New visions for the developmental assessment of infants and young children (pp. 11-26). Washington, DC: Zero to Three Press.

Greenwood, C., \& Carta, J. J. (2010). Conceptual development. In J. J. Carta, C. Greenwood, D. Walker, \& J. Buzhardt (eds.), Using IGDIs: Monitoring progress and improving intervention for infants and young children (pp. 9-22). Baltimore, MD: Brookes.

Guerette, P., Tefft, D., Furumasu, J., \& Moy, F. (1999). Development of a cognitive assessment battery for young children with physical impairments. Infant-Toddler Intervention, 9, 169-184.

Guralnick, M. J. (2000). Interdisciplinary team assessment for young children: Purposes and processes. In M. J. Guralnick (ed.), Interdisciplinary clinical assessment for young children with developmental disabilities (pp. 183-200). Baltimore, MD: Brookes. 
Hay, A., \& Brieger, A. G. (2000). Psychological assessment and the interdisciplinary team. In M. J. Guralnick (Ed.), Interdisciplinary clinical assessment of young children with developmental disabilities (pp. 3-15). Baltimore, MD: Brookes.

Hedrick, D. L., Prather, E. M., \& Tobin, A. R. (eds.). (1984). Sequenced Inventory of Communication Development (Rev. ed.): Test manual. Seattle, WA: University of Washington Press.

Iverson, J. M., \& Goldin-Meadow, S. (2005). Gesture paves the way for language development. Psychological Science, 16, 367-371.

Luyster, R. J., Kadlec, M. B., Carter, A., \& Tager-Flusberg, H. (2008). Language assessment and development in toddlers with autism spectrum disorders. Journal of Autism and Developmental Disorders, 38, 1426-1438.

McCathren, R. B., Yoder, P. J., \& Warren, S. F. (1999). The relationship between prelinguistic vocalization and later expressive vocabulary in young children with developmental delay. Journal of Speech, Language, and Hearing Research, 42, 915-924.

McDonald, E. T. (1980). Early identification and treatment of children at risk for speech development. In R. Schiefelbusch (Ed.), Nonspeech language and communication: Analysis and intervention (pp. 49-80). Baltimore, MD: University Park Press.

McLean, L., Brady, N. C., McLean, J., \& Behrens, G. A. (1999). Communication forms and functions of children and adults with severe mental retardation in community and institutional settings. Journal of Speech, Language, and Hearing Research, 42, 231-240.

Meisels, S. J., \& Atkins-Burnett, S. (2000). The elements of early childhood assessment. In J. P. Shonkoff \& S. J. Meisels (eds.), Handbook of early childhood intervention (2nd ed., pp. 231-257). New York, NY: Cambridge University Press.

Muthén, L. K., \& Muthén, B. O. (1998-2010). Mplus user's guide (6th ed.). Los Angeles, CA.

Newborg, J. (2005). Battelle Developmental Inventory (2nd ed.): Examiner's manual. Itasca, IL: Riverside.

Newborg, J., Stock, J., Wnek, L., Guidibaldi, J., \& Svinicki, J. (eds.). (1984). Battelle Developmental Inventory. Allen, TX: DLM Teaching Resources.
Oliver, B., Dale, P. S., \& Plomin, R. (2004). Verbal and nonverbal predictors of early language problems: An analysis of twins in early childhood back to infancy. Journal of Child Language, 31, 609-631.

Pueyo, R., Junque, C., Vendrell, P., Narberhaus, A., \& Segarra, D. (2008). Raven's Coloured Progressive Matrices as a measure of cognitive functioning in cerebral palsy. Journal of Intellectual Disability Research, 52, 437-445.

Raven, J., Raven, J. C., \& Court, J. H. (2003). Manual for Raven's Progressive Matrices and Vocabulary Scales. San Antonio, TX: Harcourt Assessment.

Rose, S. A., Feldman, J. F., \& Jankowski, J. J. (2009). A cognitive approach to the development of early language. Child Development, 80, 134-150.

Rose, S. A., Feldman, J. F., Jankowski, J. J., \& Van Rossem, R. (2005). Pathways from prematurity and infant abilities to later cognition. Child Development, 76, 1172-1184.

Ross, B., \& Cress, C. (2006). Comparison of standardized assessments for cognitive and receptive communication skills in young children with complex communication needs. Augmentative and Alternative Communication, 22, 100-111.

Ulvund, S. E., \& Smith, L. (1996). The predictive validity of nonverbal communicative skills in infants with perinatal hazards. Infant Behavior and Development, 19, 441-449.

Wetherby, A. M., Lonigan, C., Easterly, G., \& Stannard, L. (2002, June). First words project: Improving early identification of young children at-risk for language and reading difficulties. Poster presented at the National Association for the Education of Young Children National Institute for Early Childhood Professional Development, Chicago, IL.

Wetherby, A. M., \& Prizant, B. M. (1993). Communication and Symbolic Behavior Scales (Normed ed.): Manual. Baltimore, MD: Brookes.

Yoder, P. J., Warren, S. F., \& McCathren, R. B. (1998). Determining spoken language prognosis in children with developmental disabilities. American Journal of Speech-Language Pathology, 7, 77-87. 